\title{
ANALYTIC DOMINATION BY FRACTIONAL POWERS WITH LINEAR ESTIMATES
}

\author{
ALAN D. SLOAN
}

ABSTRACT. Conditions are given which imply the analytic domination of one operator by fractional powers of a positive selfadjoint operator. The condition $s$ involve only linear estimates rather than the usual quadratic estimates.

Let $(\mathcal{H},\|\cdot\|)$ be a normed linear space. For any linear operator $A$ on $\mathcal{H}$, let $D(A)$ denote the domain of $A$ and let $D^{\infty}(A)=\bigcap_{n=0}^{\infty} D\left(A^{n}\right)$. An element $v$ in $D^{\infty}(A)$ is called an analytic vector for $A$ if there is an $s>0$

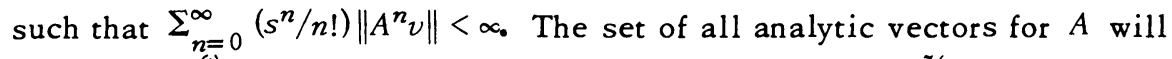
be written $D^{\omega}(A)$. Let $X$ denote another linear operator on $\mathcal{H}$. A analytically dominates $X$ if every analytic vector for $A$ is also an analytic vector for $X$, or equivalently, if $D^{\omega}(A) \subset D^{\omega}(X)$ 。

The following theorem gives conditions which guarantee analytic domination of $X$ by $A^{1 / k}, k$ a positive integer, and can be found in the Appendix of [1].

Theorem [Nelson]. Let $A$ and $X$ be everywhere defined linear operators on a normed linear space $\mathcal{H}$ such that

$$
\left\|X^{r} u\right\| \leq\|A u\|, \quad r=1,2, \ldots, k,
$$

and

$$
\left\|(\operatorname{ad} X)^{n}(A) u\right\| \leq n !\|A u\|
$$

$n=1,2, \ldots$, and $u \in \mathcal{H}$.

$$
\text { If } v \in D^{\infty}(A) \text { satisfies }
$$

$$
\left\|A^{n} v\right\| \leq M^{n}(k n) !
$$

for some $M>0$ then $v$ is an analytic vector for $X$.

Here $(\operatorname{ad} X)(A)=X A-A X$ while for $n \geq 1$,

$$
(\operatorname{ad} X)^{n+1}(A)=(\operatorname{ad} X)\left((\operatorname{ad} X)^{n}(A)\right) \text {. }
$$

Received by the editors February 11, 1974 and, in revised form, June 3, 1974. AMS (MOS) subject classifications (1970). Primary 47B25; Secondary 47 A30, $47 \mathrm{~B} 47$. 
In [2] the case $k=1$ of the above theorem was investigated with $A \geq$ $C I>0$ being a selfadjoint operator on the Hilbert space $\mathcal{H}$. It was found that the quadratic estimates (1) and (2) of the type $\pm T^{2} \leq A^{2}$ could be replaced by linear estimates $\pm T \leq A$ in the presence of some (skew-) symmetry. In this paper similar results are obtained for $k=2,3, \ldots$. Specifically we will prove the following result.

Theorem 1. Let $A$ be a selfadjoint operator on the Hilbert space $H$ with $\inf (\operatorname{spectrum}(A))>0$. Let $X: D^{\infty}(A) \rightarrow D^{\infty}(A)$. Assume

$$
\left\|A^{-1 / 2} X^{k} A^{-1 / 2} u\right\| \leq\|u\|
$$

for $k=1,2, \ldots, r$ and $u \in D^{\infty}(A)$ and

$$
\left\|A^{-1 / 2}\left((\operatorname{ad} X)^{n}(A)\right) A^{-1 / 2} u\right\| \leq n !\|u\|
$$

for $n=1,2, \ldots$ and $u \in D^{\infty}(A)$.

If $v \in D^{\infty}(A)$ satisfies

$$
\left\|A^{n} v\right\| \leq M^{n}(r n) !
$$

for some $M$ and $n=1,2, \ldots$, then $v$ is an analytic vector for $X$.

This theorem is proved with the procedure developed in [2]. First observe that if $v$ satisfies (6) then so does $A v$. Next renorm $\mathcal{H}$ with \|\| . I defined by $\|u\|=\left\|A^{-1 / 2} u\right\|$ and use Nelson's theorem to show that $A v$ is an analytic vector for $X$ relative to \|\|\|\| . Then we appeal to a result from [2] and argue that if $w$ is an analytic vector for $X$ relative to \|\|$\|$ then $A^{-1} w$ is an analytic vector for $X$ relative to \|\| . Finally we apply the last argument to $A v$ and conclude that $v=A^{-1}(A v) \in D^{\omega}(X)$.

In more detail, we have the

Proof of Theorem 1. Observe that any $v$ satisfying (6) also satisfies

$$
\sum_{n=0}^{\infty} \frac{\left((2 M)^{-1 / r}\right)^{n}}{n !}\left\|\left(A^{1 / r}\right)^{n} v\right\| \leq \sum_{k=0}^{\infty} \frac{\left((2 M)^{-1 / r}\right)^{k \cdot r}}{(k r) !}\left\|\left(A^{1 / r}\right)^{k \cdot r} v\right\| \leq \sum_{k=0}^{\infty} 2^{-k}
$$

so that $v \in D^{\omega}\left(A^{1 / r}\right)$. Conversely, if $v \in D^{\omega}\left(A^{1 / r}\right)$ then for some $t>0$, $\sum_{n=0}^{\infty}\left(t^{n} / n !\right)\left\|A^{n / r} v\right\|<\infty$ and so there is an $M<\infty$ such that $\left\|A^{n / r} v\right\| \leq n ! M^{n}$ and $\left\|A^{k} v\right\| \leq\left(M^{r}\right)^{k}(n r)$ !. Thus the set of all $v^{\prime}$ 's satisfying (6) for varying $M^{\prime}$ s is exactly $\bar{D}^{\omega}\left(A^{1 / r}\right)$. Consequently if $v$ satisfies (6) there is a $t>0$ such that $v=\exp \left(-t A^{1 / r}\right) w$ for some $w \in \mathcal{H}$. Since $A v=\exp \left(-\frac{t}{2} A^{1 / r}\right) A \exp \left(-\frac{t}{2} A^{1 / r}\right) w$, $A v$ is also in $D^{\omega}\left(A^{1 / r}\right)$ and so satisfies (6). That is, if $v$ satisfies (6) then 
there is a $Q<\infty$ such that

$$
\left\|A^{n}(A v)\right\| \leq Q^{n}(r \cdot n) !
$$

Now observe that

$$
\left\|X^{k} u\right\|=\left\|A^{-1 / 2} X^{k} u\right\|=\left\|A^{-1 / 2} X^{k} A^{-1 / 2} A^{1 / 2} u\right\| \leq\left\|A^{1 / 2} u\right\|
$$

and so

$$
\left\|X^{k} u\right\| \leq\|A u\|
$$

for $k=1,2, \ldots, r$ and $u \in D^{\infty}(A)$.

Similarly

$$
\left\|(\operatorname{ad} X)^{n}(A) u\right\| \leq n !\|\| A u \|
$$

for $n=1,2 \ldots$, and all $u$ in $D^{\infty}(A)$.

With no loss of generality we may assume $\inf (\operatorname{spectrum}(A)) \geq 1$. Then \|\|$u\|\leq\| u \|$ and any $v$ satisfying (7) will also satisfy

$$
\left\|A^{n}(A v)\right\| \mid \leq Q^{n}(r \cdot n) !
$$

With (8), (9) and (10) Nelson's theorem may be applied to the space $D^{\infty}(A)$ normed with $\|\cdot \mid\|$ yielding an $s>0$ such that

$$
\sum_{n=0}^{\infty} \frac{s^{n}}{n !}\left\|A^{-1 / 2} X^{n} A v\right\|<\infty .
$$

Combining the hypothesis of the theorem with Lemmas 3 and 4 of [2] permits the conclusion that $v=A^{-1}(A v)$ is an analytic vector for $X$ relative to \|\| . Q.E.D.

Corollary, $A^{1 / r}$ analytically dominates $X$.

Remark. If $X$ is symmetric or skew-symmetric then (4) and (5) are equivalent to $\left|\left(X^{k} u, u\right)\right| \leq(A u, u)$ and $\left|\left((\operatorname{ad} X)^{n}(A) u, u\right)\right| \leq(A u, u)$.

\section{REFERENCES}

1. R. Goodman, Analytic domination by fractional powers of a positive operator, J. Functional Analy sis 3 (1969), 246-264. MR 39 \#801.

2. A. Sloan, Analytic domination with quadratic form type estimates and nondegeneracy of ground states in quantum field theory, Trans. Amer. Math. Soc. 194 (1974), 325-336.

SCHOOL OF MATHEMATICS, GEORGIA INSTITUTE OF TECHNOLOGY, ATLANTA, GEORGIA 30332 